\title{
Role of alternance symmetry in magnetoconductance
}

\author{
Josep Planelles* and Juan I. Climente \\ Departament de Química Física i Analítica, Universitat Jaume I, E-12080, Castelló, Spain \\ (Received 22 January 2014; revised manuscript received 8 April 2014; published 28 April 2014)
}

\begin{abstract}
We show that the direction of coherent electron transport across a cyclic system of quantum dots or a cyclic molecule can be modulated by an external magnetic field if the cycle has an odd number of hopping sites, but the transport becomes completely symmetric if the number is even. These contrasting behaviors, which remain in the case of interacting electrons, are a consequence of the absence or presence of alternance symmetry in the system. These findings are relevant for the design of nanocircuits based on coupled quantum dots or molecular junctions.
\end{abstract}

\section{INTRODUCTION}

Electron transport across cyclic quantum dot molecules is a subject of active research in condensed matter physics, where "artificial molecules" made of tunnel-coupled quantum dots with looped structure can be fabricated [1,2]. The interest of these systems follows from the quantum-mechanical interference of electronic paths, which gives rise to a variety of physical phenomena. In recent years, much attention has been paid to triple-dot structures, owing to their potential for the development of spin qubits [3]. Four-dot structures have also been proposed for spin qubits, because they constitute the smallest loop enabling decoherence-free subspaces [4,5].

In parallel, there is also a current endeavor to develop molecular wires and molecular electronics based on singlemolecule junctions [6-8]. Here there is also interest in conjugated cyclic molecules, as their multiply-connected topology is expected to enable conductance modulation by means of the quantum interference processes [9-13].

Clear manifestations of the topology of cyclic systems are expected in their magnetoconductance, as the magnetic flux enclosed by the delocalized electron translates into an Aharonov-Bohm (AB) phase [14] whose consequences on the electronic transport have been discussed in the literature, be it for aromatic hydrocarbons $[10,11,15]$, for coupled quantum dots [1,16-19], or for ring-shaped nanostructures [20-22].

While the role of spatial and spin symmetries in the transport and magnetotransport of cyclic systems has been thoroughly investigated $[13,16,19,23,24]$, much less is known about the role of the so-called alternance symmetry. From the early times of quantum chemistry, alternance symmetry has proven very useful in predicting the properties of conjugated hydrocarbons. This topology-related symmetry classifies the conjugated hydrocarbons into two nonoverlapping groups: alternant or nonalternant [25]. The idea is to divide all carbons in a molecule into two sets, one marked with stars $(\star)$ and the other with circles $(\circ)$. The system is alternant if it is possible to place stars (circles) on alternating carbons, with no two stars (circles) adjacent. Thus, hydrocarbons with odd-membered cycles are nonalternant, while linear structures and evenmembered cycles are alternant. The bipartite nature of alternant systems gives rise to a particle-hole symmetry in the single

\footnotetext{
*josep.planelles@uji.es; http://quimicaquantica.uji.es/
}

particle energy spectrum which is missing in nonalternant systems. As a result, molecules of the each kind share features of the electronic structure whose impact on the reactivity and spectroscopy have been long recognized [26-29].

In this work, we study single-electron transport across planar cyclic systems subject to an external axial magnetic field. The goal is to gain understanding of the role of alternance symmetry on such transport. To this end, we model both alternant and nonalternant systems using a Hubbard Hamiltonian. The cyclic systems are coupled to one input and two output channels, forming a three-terminal device. When the system is nonalternant, the magnetic field is shown to modulate the direction of electron transport, favoring the transfer probability through one of the output channels. When the system is alternant, by contrast, the transport is completely symmetric through both output channels. An interpretation is provided for these results by analyzing the alternance symmetry in the Hamiltonian. The influence of excess interacting electrons in the molecule is also investigated. It is found that the role of alternance symmetry holds in the few-electron case.

The paper is organized as follows. In Sec. II we introduce the theoretical model. In Sec. III we describe the numerical simulations of electron magnetotransport in both alternant and nonalternant molecules. In Sec. IV we discuss how these results may relate to either molecular devices using aromatic molecules or solid state quantum dot circuits. Finally, in Sec. V we give conclusions.

\section{THEORETICAL CONSIDERATIONS}

\section{A. Hamiltonian and time evolution}

In order to describe our system of interacting fermions we use a Hubbard Hamiltonian. The Hamiltonian, built on a nearest-neighbor tight-binding (TB) formalism and including a perpendicular magnetic field, reads

$$
\begin{aligned}
H= & \sum_{i} \epsilon_{i} c_{i}^{\dagger} c_{i}+\sum_{\langle i j\rangle}\left[t_{i j}^{0} e^{i \theta_{i j}} c_{i}^{\dagger} c_{j}+\text { H.c. }\right] \\
& +U \sum_{i}\left(n_{i}^{2}-n_{i}\right) / 2,
\end{aligned}
$$

where $c_{i}^{\dagger}\left(c_{i}\right)$ denotes the creation (annihilation) operator of the site $i, n_{i}$ is the number operator, and $\epsilon_{i}$ is the on-site potential at the $i$ th site that we set equal to zero unless otherwise 
indicated. $t_{i j}^{0}$ is the zero-field tunneling parameter between the nearest-neighbor sites $i$ and $j$, and $\theta_{i j}$ is the Peierls phase given by $\theta_{i j}=\left(2 \pi / \Phi_{0}\right) \int_{i}^{j} \mathbf{A} \cdot d \mathbf{l}$, with $\Phi_{0}=h c / e$ being the magnetic flux quantum and $\mathbf{A}$ the vector potential [30]. This vector potential for a uniform axial magnetic field, employing the symmetric gauge, results in $\mathbf{A}=\frac{B}{2}(-y, x, 0), B$ being the magnetic field strength. Finally, $U$ stands for the Hubbard repulsion parameter.

Obviously, the system dynamics does not depend on the selected gauge for the potential vector and the selected origin of coordinates. We thus set the origin of coordinates at the center of the cyclic molecule in our three-terminal devices. Since the molecule can be described by a regular polygon, it is straightforward to show that all nearest-neighbor $t_{i j}$ tunneling parameters connecting two neighbor polygon vertices are the same, once the index rotation sense is fixed. Note that $t_{j i}=t_{i j}^{*}$, and again, the dynamics of the system does not depend on the selected sense of rotation. Further, since the Hubbard Hamiltonian (1) only depends on the topology, one may assume - without loss of generality - that the input and output channels (or leads) are arranged radially from the vertices of the polygon. Because the in-plane coordinates $x$ and $y$ along a radial line are related by $y=a x$, the Peierls phase between consecutive sites $i$ and $j$ of such line becomes zero, as $\int_{i}^{j} \mathbf{A} \cdot d \mathbf{l}=\frac{B}{2} \int_{i}^{j}(-a x, x, 0) \cdot(d x, a d x, 0)=0$. In other words, the tunneling parameter between two lead sites or between a polygon site and a neighbor lead site can be set to $t_{i j}^{0}$, regardless of the field and the actual geometry.

To solve Hamiltonian (1) for $N$ electrons, the Hamiltonian is expanded onto the complete full configuration interaction (FCI) space containing $\Omega=\left(\begin{array}{c}2 K \\ N\end{array}\right)$ Slater determinants $D_{i}(1,2, \ldots, N)=\mid \chi_{i}(1) \sigma_{i}(1) * \chi_{j}(2) \sigma_{j}(2) * \cdots *$ $\chi_{k}(N) \sigma_{k}(N) \mid$, where $K$ is the number of independent particle functions. $K$ is also the number of sites in the system, as our TB model considers a single independent particle orbital $\chi_{i}$ centered at site $i$. The wave function is then

$$
\Psi(1,2, \ldots, N)=\sum_{i}^{\Omega} c_{i}(t) D_{i}(1,2, \ldots, N) .
$$

The expectation value of the density operator $\hat{\rho}(\mathbf{r})=$ $\sum_{i}^{N} \delta\left(\mathbf{r}-\mathbf{r}_{i}\right)$ results in

$$
\langle\Psi|\hat{\rho}| \Psi\rangle=\sum_{i j}^{N} c_{i}(t)^{*} c_{j}(t)\left\langle D_{i}|\hat{\rho}(\mathbf{r})| D_{j}\right\rangle,
$$

and the population of site $a$ is

$$
\langle\Psi|\hat{\rho}| \Psi\rangle_{a}=\sum_{i}^{N}\left|c_{i}(t)\right|^{2}\left(\delta_{a, \alpha_{i}}+\delta_{a, \beta_{i}}\right),
$$

where $\delta_{a, \alpha_{i}}\left(\delta_{a, \beta_{i}}\right)$ is zero unless $\chi_{a}$ is present in $D_{i}$ with spin $\alpha(\beta)$.

The time-dependent Schrödinger equation for Hamiltonian (1) projected on the wave function (2) can be written in atomic units as

$$
i \frac{d}{d t} \mathbb{C}=\mathbb{H} \mathbb{C}
$$

which is equivalent to

$$
\mathbb{C}(t)=\mathbb{U}(t) \mathbb{C}(0),
$$

where $\mathbb{U}(t)=\exp (-i t \mathbb{H})$ is the time evolution operator. Note that Eq. (6) is equivalent to Eq. (5) because we do not introduce time-dependent parameters in the Hamiltonian [31].

It is convenient to define effective tunneling and time scales as follows. We factorize the tunneling parameter $t^{0}$ in the Hamiltonian: $H=t^{0} H_{0}$, where $H_{0}$ is now the Hamiltonian $H$ in effective units (e.u.) Next, we replace $t * t^{0} \rightarrow t$ in $\mathbb{U}(t)$, so that time is now given in e.u. Thus, we melt the dependence of the time-evolution operator in a single time-like parameter $t$ (e.u.) that makes changes in the system within the length scale, as we will see in Sec. III.

\section{B. Alternance symmetry}

The alternance symmetry is closely related to the topological properties of the TB approximation. This symmetry is related to the possibility to divide centers into two disjoints sets $C^{\star}$ and $C^{\circ}$ in such a way that any center of one set (say $C^{\star}$ ) can only tunnel to centers of the other set $\left(C^{\circ}\right)$ and vice versa, so that the complete system has a bipartite graph structure. Although strictly speaking this symmetry-and the ensuing invariance properties-is only present when the system is described by an approximate model Hamiltonian, it may prove to be of great value in the classification of corresponding eigenstates, and the resulting selection rules in an interpretation of various spectral characteristics of the system. In other words, it has an indisputable physical origin. The importance of the approximate selection rules which result from this symmetry has been long recognized in one-photon absorption and emission spectroscopy [26], magnetic circular dichroism [27], two-photon absorption spectroscopy [28], etc.

Alternance symmetry was first studied in the independent particle Hückel Hamiltonians [33], and later for the interacting particles Pariser-Parr-Pople (PPP) Hamiltonian [26,34], these papers being complemented by others in a proper assessment of the meaning, scope, connections and relevance of this symmetry (see, e.g,. Refs. [35] and [36]). The Hubbard Hamiltonian [37] can be considered an approximate PPP Hamiltonian where some minor terms in the repulsion part of the Hamiltonian have been neglected. In turn, TB can be considered an approximate PPP Hamiltonian where all repulsion terms are neglected. Since the alternance symmetry is related to the topology, all three Hamiltonians display the symmetry.

Alternance symmetry implies one-electron pairing properties. Namely, the independent particle eigenfunctions $\left|a_{i}\right\rangle$ and their corresponding energies $\varepsilon\left(\left|a_{i}\right\rangle\right)$ in an alternant system are paired in such a way that for each eigenfunction $\left|a_{i}\right\rangle=$ $\sum_{\mu} c_{i \mu}|\mu\rangle$, where $|\mu\rangle$ are the one-site TB functions and $c_{i \mu}$ is the coefficient of the expansion of $\left|a_{i}\right\rangle$ in terms of the basis set $\{|\mu\rangle\}$, we can associate the alternant conjugate eigenfunction $\left|\tilde{a}_{i}\right\rangle=\sum_{\mu} \tilde{c}_{i \mu}|\mu\rangle$ such that $\tilde{c}_{i \mu}=c_{i \mu}$ if $\mu \in C^{\circ}$ and $\tilde{c}_{i \mu}=$ $-c_{i \mu}$ if $\mu \in C^{\star}$. In addition, $\varepsilon\left(\left|\tilde{a}_{i}\right\rangle\right)=-\varepsilon\left(\left|a_{i}\right\rangle\right)+$ constant. Interestingly, since this symmetry is related to the topological properties of the TB it can be modulated by the magnetic field, because it comes into the Hamiltonian as a phase in the tunneling integral. 

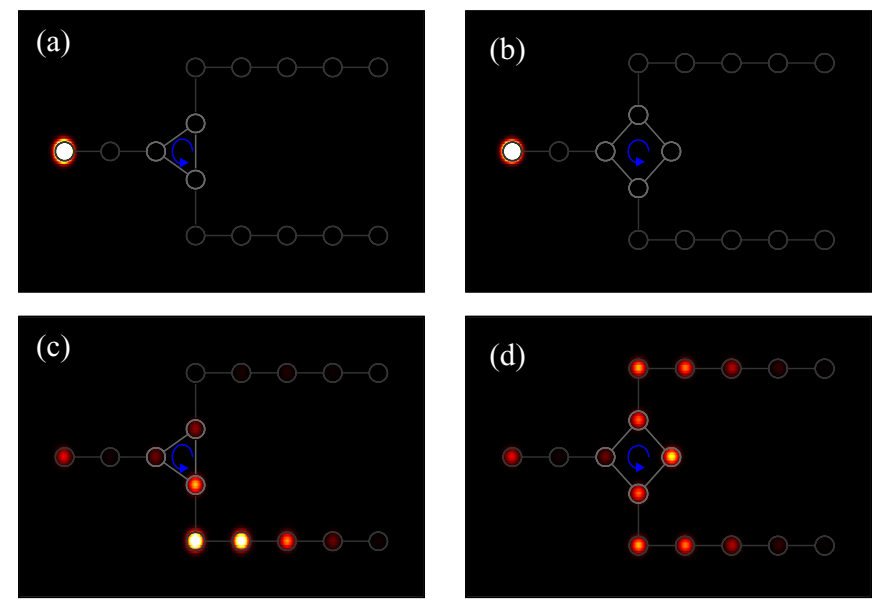

FIG. 1. (Color online) Electron density at different times in three-terminal devices under a magnetic flux of $\phi=0.25 \phi_{0}$. (a),(c) Triangular system at $t=0$ and $t=3$ e.u., respectively. The magnetic flux favors transport through the lower output channel. (b),(d) Rhombic system at $t=0$ and $t=3$ e.u., respectively. The transport is symmetric in spite of the magnetic flux.

\section{RESULTS}

\section{A. Single-electron transport}

We start by investigating electron transport across a triangular cycle. This is the simplest nonalternant system, its behavior being representative of that found in other nonalternant cycles. A perpendicular magnetic field is applied which gives rise to a tunneling parameter $t_{i j}^{0}=t^{0} e^{i \frac{2 \pi}{3} \frac{\Phi}{\Phi_{0}}}$, where $i$ and $j$ are any two nearest-neighbor sites of the triangle. The cyclic system is attached to one input and two output channels arranged symmetrically, with one electron initially prepared in the first site of the incoming channel, as schematically depicted in Fig. 1(a). For the actual calculations, the output channels have ten sites each. We assume all the sites have the same potential, $\epsilon_{i}=\epsilon=0$, and the hopping parameter is $t^{0}=-1$ e.u.

With evolving time, the electron travels along the input channel and reaches the triangular system. At this point, the electron feels the magnetic flux favoring a given sense of rotation (represented by the blue curved arrow in the figure). For $\phi=0.25 \phi_{0}$, the flux strongly favors electron transfer into the lower channel. This can be seen in Fig. 1(c), which shows the electron density at a finite time when the electron leaves the cycle. If a flux of $\phi=-0.25 \phi_{0}$ was used, the transport would take place through the upper channel instead.

For comparison, we next investigate electron transport across a rhombic cycle. Again, this is the simplest alternant system, and it is representative of more complex alternant cycles. The tunneling parameter is now $t_{i j}^{0}=t^{0} e^{i \frac{2 \pi}{4} \frac{\Phi}{\Phi_{0}}}$. Figure 1(b) shows the initial setup and Fig. 1(d) the corresponding electron density at a finite time. Clearly, the electron transport is in sharp contrast to that of the triangle, the transfer probability through upper and lower channels now being identical.

Three-terminal devices have been employed by several groups both in mesoscopic and molecular systems (for reviews see, e.g., Refs. [7] and [11]), and the possibility to switch the direction of electron transport magnetically in such systems

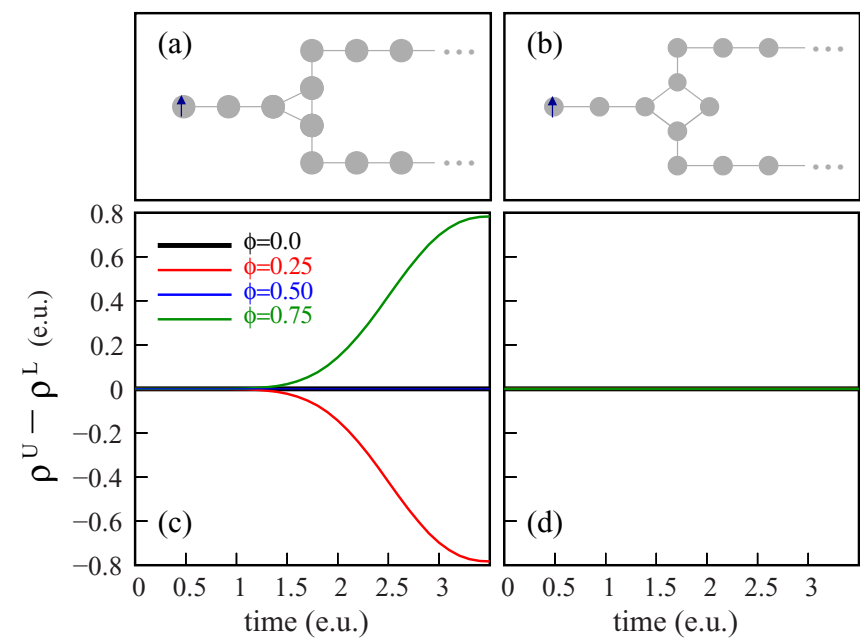

FIG. 2. (Color online) (a),(b) Schematic of the systems under study at $t=0$, and (c),(d) corresponding differences between electron density in the upper and lower channel as a function of time for different magnetic fluxes. The fluxes are in flux quantum $\left(\phi_{0}\right)$ units.

had been predicted by Hod et al., who further discussed the convenience of the magnetic modulation as an alternative to electrical manipulation $[10,11]$. In this context, Fig. 1 reveals that the magnetic control of the electron transport can only be achieved in nonalternant systems, thus establishing a critical parameter in the design of molecular junctions. This is the central finding of this work. In what follows we will gain a deeper understanding of this phenomenon and discuss its implications.

In order to generalize the result of Fig. 1, we next compare electron transport in the triangular and rhombic systems for several values of the magnetic flux $\phi$. The systems under study are represented schematically in Figs. 2(a) and 2(b), which show the setups at the initial time $t=0$. To monitor the dynamics, we compute the electron density in the upper and lower channels as $\rho_{i}=\sum_{a}\langle\Psi|\hat{\rho}| \Psi\rangle_{a}$, where $i=U, L$ denotes the channel and $a$ runs over all the sites of the channel. Note that for long times, when the charge density has left the cyclic system, $\rho_{i}$ can be identified with the transfer probability through the $i$ th channel $T_{i}$. The conductance is proportional to this magnitude [38].

Figure 2(c) shows the difference between the density in the upper and lower channels as a function of time for the triangular cycle. In the absence of magnetic flux $\phi=0 \phi_{0}$, the transport is symmetric through both output channels. Switching on a positive magnetic flux $\phi=0.25 \phi_{0}$, most of the transport takes place along the lower channel, as anticipated in Fig. 1(c). However, for $\phi=0.5 \phi_{0}$ the transport becomes again symmetric, and for $\phi=0.75 \phi_{0}$ the behavior reverses, with transport taking place mostly along the upper channel.

The influence on the magnetic flux on the sense of electron rotation can be understood easily by considering the triangle as a closed system. In the basis of atomic sites and using atomic units, the resulting eigenstates are

$$
\Psi_{m}=\left(\begin{array}{c}
e^{i(m+\phi) 0} \\
e^{i(m+\phi) 2 \pi / 3} \\
e^{-i(m+\phi) 2 \pi / 3}
\end{array}\right),
$$


associated to energies $\varepsilon_{m}=\varepsilon+2 t^{0} \cos \left(\frac{2 \pi(m+\phi)}{3}\right)$, with $m=$ $0 \pm 1$. Although in triangles (finite-edges polygons in general) the angular momentum $L_{z}$ is not a constant of motion, we may still relate $m>0(m<0)$ to anticlockwise (clockwise) rotation [39]. Therefore, when $\phi=0, \Psi_{1}$ and $\Psi_{-1}$ become degenerate, so that this energy level does not have a preferred sense of rotation. The same happens to the nondegenerated $m=0$ level. This is the underlying physical reason for the symmetric evolution on time of a state in which the electron is initially set at one site of the triangle that evolves symmetrically to the other two sites.

Switching on the magnetic field introduces an effective angular momentum $m_{\phi}=(m+\phi)$ and lifts degeneracies, resulting in nonzero angular momenta and a favored direction of rotation. A special situation is found at half-integer values of the flux. Then degeneracy between states with opposite effective angular momenta is found (e.g., $m_{\phi}=0.5$ and $m_{\phi}=$ -0.5 at $\phi=0.5$ ), and again there is no net angular momentum. As a consequence, transport is again symmetric in both senses. In short, the direction of electron transport in the triangle is determined by the combined effect of angular momenta and magnetic flux. It is symmetric when $\phi=k / 2$ with $k$ integer, and asymmetric otherwise. Anticlockwise (clockwise) rotation is favored for $k / 2<\phi<(k+1) / 2$ when $k$ is even (odd), with a maximum at $\phi=k / 4$.

We now investigate transport through a rhombic cycle, Fig. 2(d). The striking result is that, contrary to the case of the triangle, the dynamics is completely symmetric for any value of the magnetic flux. In other words, using a cycle with an even number of sites removes the magnetic modulation of electron transport direction. This is a consequence of the alternance symmetry and its relation to time-reversal symmetry, as we show next [40].

Let us consider a TB alternant system, in the presence of a magnetic field. We set the origin of energies at the center of the energy spectrum so that $\epsilon_{j}=-\epsilon_{K-j}$, where $K$ is the number of sites in the system and hence also the dimension of the basis set. We can define the operator $\hat{T}$ acting on the independent particle functions $\chi_{j}$ of the system:

$$
\hat{T} \chi_{j} e^{-i \epsilon_{j} t}=\chi_{K-j} e^{i \epsilon_{j} t}=\chi_{K-j} e^{-i \epsilon_{K-j} t} .
$$

This operator does not commute with the Hamiltonian, but rather anticommutes with it, $\{\hat{T}, H\}=\hat{T} H+H \hat{T}=$ 0 . Then it represents a symmetry of the time-dependent Schrödinger equation, which transforms $i d / d t \Psi_{j}=H \Psi_{j}$ into $i d / d t \Psi_{K-j}=H \Psi_{K-j}$. This allows us to define a partition of the wave function space into a symmetric $\Psi_{+}=\Psi_{j}+\Psi_{K-j}$ and an antisymmetric $\Psi_{-}=\Psi_{j}-\Psi_{K-j}$ part. Since $\Psi_{j}=\sum_{i}^{\left\{C^{\circ}\right\}} a_{i} \chi_{i}^{\circ}+\sum_{j}^{\left\{C^{\star}\right\}} b_{j} \chi_{j}^{\star}$ and $\Psi_{K-j}=$ $\sum_{i}^{\left\{C^{\circ}\right\}} a_{i} \chi_{i}^{\circ}-\sum_{j}^{\left\{C^{\star}\right\}} b_{j} \chi_{j}^{\star}$, the functions $\Psi_{+} / \Psi_{-}$have only nonzero coefficients at the sites $C^{\circ} / C^{\star}$. Thus, preparing the electron initially in a $C^{\circ}\left(C^{\star}\right)$ site (or several sites of the same kind) means the system is in a state defined by $\Psi_{+}(0)\left[\Psi_{-}(0)\right]$. Because $\epsilon_{j}=-\epsilon_{K-j}$, the system will experience, as time runs, a synchronic decrease of population in the sites $C^{\circ}\left(C^{\star}\right)$ and a simultaneous increase of it at the partner sites $C^{\star}\left(C^{\circ}\right)$. Note that this will happen whether or not an axial magnetic field, favoring a given sense of electronic circulation, acts on the system.

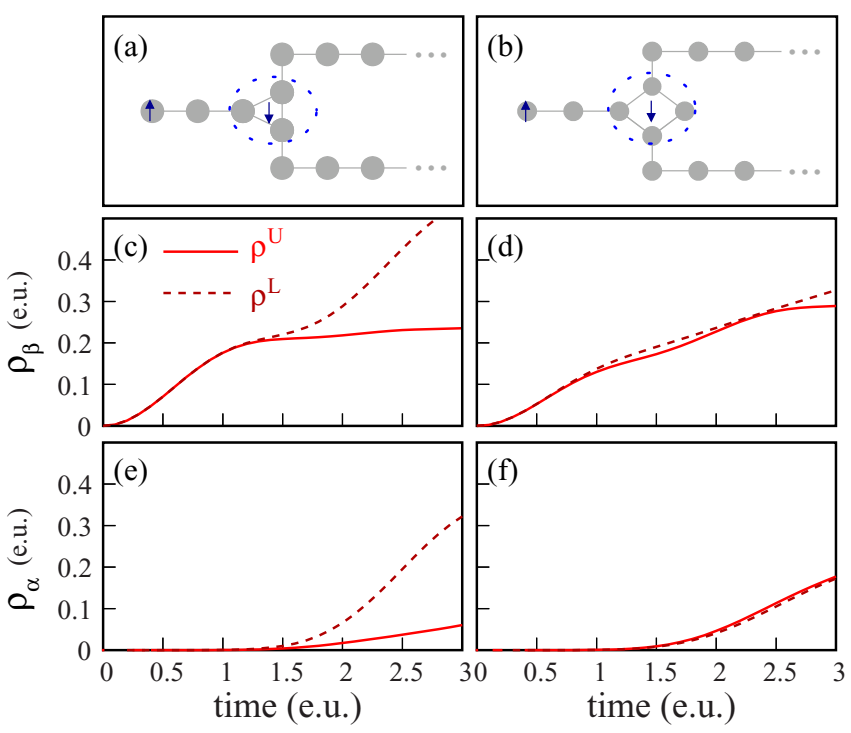

FIG. 3. (Color online) (a)-(b) Schematic of the systems under study at $t=0$. (c)-(d) Corresponding differences between spin $\beta$ electron density in the upper and lower channel as a function of time; (e)-(f) same but for spin $\alpha$ electron. In all cases $\phi=0.25 \phi_{0}$. Note that $\rho_{\alpha}$ monitors the electron initially localized in the first site of the incoming channel, while $\rho_{\beta}$ does so for the electron initially delocalized over the cyclic system.

\section{B. Few-electron transport}

We next investigate the effect of electron-electron repulsion on the magnetotransport of our three-terminal devices. To this end we add a resident electron delocalized over the cyclic system, as plotted in Figs. 3(a)-3(b). The Hubbard repulsion parameter is set to $U=10 t^{0}$, the ratio $U /\left|t^{0}\right|=10$ thus being of the same order of magnitude as that employed in molecular systems [41] and quantum dots [16].

The resident electron has opposite $\operatorname{spin}(\beta)$ to that of the incoming electron $(\alpha)$. This allows us to track their time evolutions separately by plotting the spin polarized electron densities. Thus, for the triangular system, Fig. 3(c) shows the time evolution of the resident electron $(\operatorname{spin} \beta$ electron density), while Fig. 3(e) does so for the incoming one (spin $\alpha$ density). We have set $\phi=0.25 \phi_{0}$. One can see that the resident electron spreads into the output channels symmetrically for times $t<1.5$ e.u. This is in spite of the system being nonalternant. The reason is that the electron is initially delocalized in all three sites of the triangle, so that the magnetic-field-induced rotation favors transport equally through all three channels, much as in a sprinkler. For longer times, however, transport through the lower channel is suddenly favored. It is worth noting that this coincides with the arrival of the spin $\alpha$ electron, see Fig. 3(e). This is because the spin $\beta$ density, first scattered across the input channel, bounces back when it meets the spin $\alpha$ electron owing to Coulomb repulsion. It then reenters the system core and travels as in the single-electron case described in the previous subsection, i.e. mostly through the lower channel. The incoming $\alpha$ electron also follows this path.

For the rhombic system, Fig. 3(f) shows the incoming electron travels symmetrically, as in the single-electron system. 

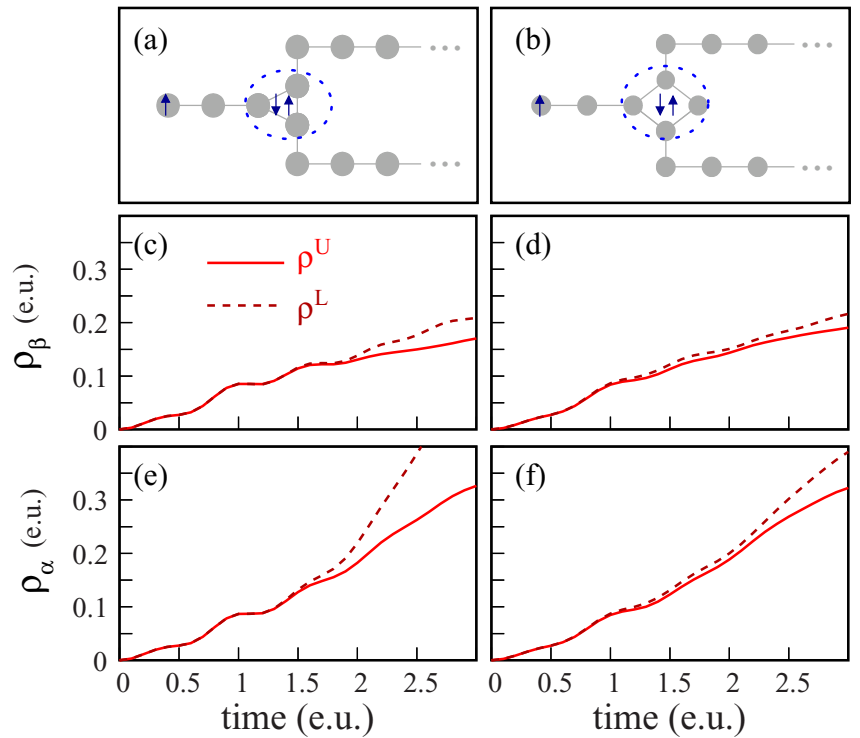

FIG. 4. (Color online) Same as Fig. 3 but with two resident electrons forming a singlet.

Instead, Fig. 3(d) shows that the resident electron transport is no longer exactly symmetric. This is because the resident electron initially occupies both $C^{\circ}$ and $C^{\star}$ sites, so its wave function cannot be described by $\Psi_{+}$or $\Psi_{-}$alone. In other words, alternance symmetry is broken, and hence symmetric transport is no longer granted.

Similar findings are obtained if the system contains two spin-paired electrons. Figure 4 shows the corresponding results. For short times $(t<1.5$ e.u.), transport is symmetric through both output channels owing to the sprinkler-like effect. By comparing $\rho_{\alpha}$ and $\rho_{\beta}$, no spin polarized transport is observed in this regime. For longer times, when the incoming electron arrives, the transfer probability increases in the lower lead.

We conclude from this subsection that the behavior of interacting electrons essentially follows an independent particle scheme, except for the presence of the Coulomb hole. The reason is that for a ratio $U /\left|t^{0}\right|=10$ double-occupancy states are at much higher energy than single-occupancy ones. Then the conservation of the average energy $\langle E\rangle$ along time only allows minute contributions of these states in $\Psi(t)$, so the system evolution resembles that of independent particles. In setups with lower $U /\left|t^{0}\right|$ ratio, Coulomb correlations may play a role, but in the limit of low $U /\left|t^{0}\right|$ ratio, electronelectron interactions become negligible and we are again in the independent particle scheme. Thus, in most cases the role of alternance symmetry on the electron transport is captured by the single-electron picture.

\section{DISCUSSION}

In this section we briefly discuss some implications of alternance symmetry on the electron magnetotransport through existing or potentially interesting molecular and mesoscopic systems.

\section{A. Molecular junctions}

As mentioned earlier, while the role of spatial symmetry in molecular junctions is under active research [13,23,24], that of alternance symmetry has been overlooked so far. As shown in the previous section, alternance symmetry is critical in determining the magnetoconductance of cyclic systems. In particular, its absence (presence) enables (disables) magnetic control of the electron transport direction. This kind of control has been proposed as an alternative to electric control [11]. In the case of molecules, the main handicap is that very strong magnetic fields are required to achieve significant flux piercing the small area of usual molecules, such as benzene. This problem can be overcome by using macromolecules with larger areas. Several systems are suited to this end, including quantum corrals made of metal atoms [42], polyaromatic hydrocarbons [15], colloidal graphene quantum dots [43], or nanographene rings. As a matter of fact, it has been recently recognized that the latter structures often present defects in the form of odd-membered rings [44]. This clearly breaks the alternance symmetry, and the ensuing consequences on the transport should be born in mind.

Hod and co-workers have proposed a magnetoresistance logic gate based on a three-terminal device containing a macrocycle composed of 48 benzene rings [10]. The operating principle relied on the asymmetric transport through the two output leads induced by an external magnetic field. It is worth noting that they expect asymmetric transport in spite of having considered an even-membered ring. The reason is that the the macromolecule is attached to gold leads. In our work we have assumed that all sites have the same potential $\epsilon$. Introducing sites with different energy, such as carbon and gold atoms, renders the energy spectrum asymmetric with respect to its center, hence breaking the symmetric transport. This effect is strong when the heteroatoms are in contact with the cycle and gradually fades when they are away from it, as can be seen in Fig. 5 for the systems in the insets. The figure shows the electron density reaching the upper and lower channels when the electron is initially injected in a heteroatom which is one site (left panels) and twenty sites (right panels) away from the alternant cycle. Typical heteroatoms in conjugated

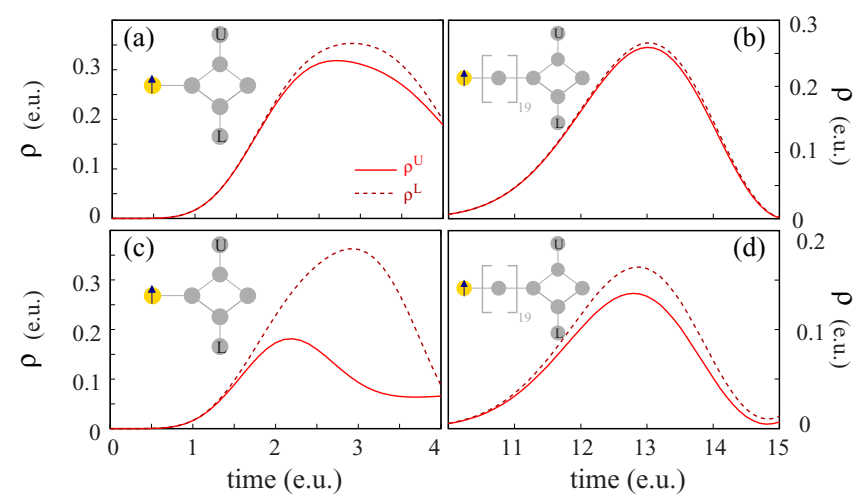

FIG. 5. (Color online) Electron density in the upper and lower channel as a function of time at $\phi=0.25 \phi_{0}$. (a),(b) The injected electron is in a heteroatom with $\Delta \epsilon=0.1 t^{0}$. (c),(d) The heteroatom has $\Delta \epsilon=t^{0}$. The insets show the structure under study. Left (right) column: the heteroatom is next to (20 sites away from) to the cycle. 
molecules have energies which differ from those of carbon atoms by $\Delta \epsilon \approx 0.5-2 t^{0}$ [45]. We then consider two possible orders of magnitude, $\Delta \epsilon=0.1 t^{0}$ (top panels) and $\Delta \epsilon=1.0 t^{0}$ (bottom panels). When the heteroatom is next to the cycle [Figs. 5(a) and 5(c)], one observes asymmetry not only in the amount of density reaching the upper and lower channels, but also in the time this occurs. Clearly, the asymmetry is more pronounced in Fig. 5(c), indicating that the nature of the heteroatom (and hence $\Delta \epsilon$ ) is critical in determining the extent of the asymmetry. Still, when the heteroatom is away from the cycle [Figs. 5(b) and 5(d)] the asymmetry is visibly reduced. This suggests that, even in the presence of heteroatoms, nearly symmetric transport associated with alternance symmetry can be expected if the input channel is long enough.

As opposed to the potential of the sites $\epsilon$, varying the tunneling parameter $t_{i j}^{0}$ of the leads with respect to that of the cycle does not induce any asymmetric transport. As an illustration of the applications of alternance symmetry, with the above considerations, one could envisage a molecular device with selective electron transport for photocatalysis or photocurrent generation. Electrons are generated in a photoactive group at the far end of the input chain. Next they travel until a cyclic macromolecule which is subject to a constant magnetic field. The molecule has two additional (output) chains contacting the electrodes. If the molecule is nonalternant, the magnetic field will drive electron transport preferentially through one of the chains. If it is alternant, the transport will be symmetric instead, although one can easily switch to nonalternant, e.g., by chemical substitution of the aromatic cycles.

\section{B. Coupled quantum dots}

Contrary to molecular systems, the magnetoconductance of coupled quantum dot systems has been thoroughly investigated theoretically and experimentally. Several studies have dealt with triangular triple-dot structures [1,16-19], but squared four-dot cycles have also been proposed $[5,46]$. Much of the interest in these systems relied on the influence of the $A B$ effect on the electron transport.

The large magnetic fluxes that can be achieved in these systems make them ideal to experimentally test the role of alternance symmetry described in this paper. The main difficulty will be to produce truly alternant systems. The chemical potential of the leads can be set to match that of the quantum dots, so that all sites have the same energy, while electrostatic gates can be used to minimize the energy difference between the dots, which are otherwise different owing to size and composition inhomogeneities. Yet, electrostatic fluctuations may play a role [47].

We close by noting that the magnetotransport we report here is a pure quantum mechanical effect, derived from the nontrivial $\mathrm{AB}$ phase factor introduced by the magnetic flux piercing the cyclic system. In finite-width quantum dots, the magnetic field also pierces the dot itself, and an additional asymmetry in the transport may arise from the Lorentz force acting on the electrons, as noticed in quantum rings [22].

\section{CONCLUSIONS}

In conclusion, using a Hubbard model we have shown that the sense of electron transport across a cyclic system can be governed by magnetic fields only if it is nonalternant. The presence of alternance symmetry imposes symmetric transport in all directions. This result is independent of the magnetic field value and it holds both for single-electron and few-interacting electron systems. We then argue that this topological symmetry is a critical parameter in the design of atomic or mesoscopic molecular junctions for magnetoconductance devices.

\section{ACKNOWLEDGMENTS}

Support from MICINN project CTQ2011-27324 and UJIBancaixa project P1-1B2011-01 is acknowledged.
[1] L. Gaudreau, A. S. Sachrajda, S. Studenikin, A. Kam, F. Delgado, Y. P. Shim, M. Korkusinski, and P. Hawrylak, Phys. Rev. B 80, 075415 (2009).

[2] E. Taranko, M. Wiertel, and R. Taranko, J. Appl. Phys. 111, 023711 (2012), and references therein.

[3] L. Gaudreau, G. Granger, A. Kam, G. C. Aers, S. A. Studenikin, P. Zawadzki, M. Pioro-Ladriere, Z. R. Wasilewski, and A. S. Sachrajda, Nat. Phys. 8, 54 (2012).

[4] D. A. Lidar, I. L. Chuang, and K. B. Whaley, Phys. Rev. Lett. 81, 2594 (1998).

[5] V. W. Scarola, K. Park, and S. Das Sarma, Phys. Rev. Lett. 93, 120503 (2004).

[6] A. Nitzan and M. A. Ratner, Science 300, 1384 (2003).

[7] N. J. Tao, Nature Nanotechnol. 1, 173 (2006).

[8] H. Song, M. A. Reed, and T. Lee, Adv. Mater. 23, 1583 (2011).

[9] C. Liu, D. Walter, D. Neuhauser, and R. Baer, J. Am. Chem. Soc. 125, 13936 (2003).
[10] O. Hod, R. Baer, and E. Rabani, J. Am. Chem. Soc. 127, 1648 (2005).

[11] O. Hod, R. Baer, and E. Rabani, J. Phys.: Condens. Matter 20, 383201 (2008).

[12] T. Hansen, G. C. Solomon, D. Q. Andrews, and M. A. Ratner, J. Chem. Phys. 131, 194704 (2009).

[13] T. Markussen, R. Stadler, and K. S. Thygesen, Nano Lett. 10, 4260 (2010).

[14] Y. Aharonov and D. Bohm, Phys. Rev. 115, 485 (1959).

[15] M. Mardaani and H. Rabani, J. Magn. Magn. Mater. 331, 28 (2013).

[16] F. Delgado, Y. P. Shim, M. Korkusinski, L. Gaudreau, S. A. Studenikin, A. S. Sachrajda, and P. Hawrylak, Phys. Rev. Lett. 101, 226810 (2008).

[17] Z. T. Jiang and Q. F. Sun, J. Phys.: Condens. Matter 19, 156213 (2007).

[18] C. Emary, Phys. Rev. B 76, 245319 (2007). 
[19] T. Kuzmenko, K. Kikoin, and Y. Avishai, Phys. Rev. Lett. 96, 046601 (2006).

[20] R. A. Webb, S. Washburn, C. P. Umbach, and R. B. Laibowitz, Phys. Rev. Lett. 54, 2696 (1985); G. Timp, A. M. Chang, J. E. Cunningham, T. Y. Chang, P. Mankiewich, R. Behringer, and R. E. Howard, ibid. 58, 2814 (1987).

[21] T. Chwiej and B. Szafran, J. Phys.: Condens. Matter 25, 155802 (2013); M. Ijäs and A. Harju, Phys. Rev. B 85, 235120 (2012).

[22] B. Szafran and F. M. Peeters, Europhys. Lett. 70, 810 (2005).

[23] M. Taniguchi, M. Tsutsui, R. Mogi, T. Sugawara, Y. Tsuji, K. Yoshizawa, and T. Kawai, J. Am. Chem. Soc. 133, 11426 (2011).

[24] E. Lörtscher, ChemPhysChem 12, 2887 (2011).

[25] M. Orchin, R. S. Macomber, A. R. Pinhas, and R. M. Wilson, The Vocabulary And Concepts of Organic Chemistry (John Wiley \& Sons, New Jersey, 2005).

[26] R. Pariser, J. Chem. Phys. 24, 250 (1956).

[27] J. Michl, J. Chem. Phys. 61, 4270 (1974); R. J. van der Wal and P. J. Zandstra, ibid. 64, 2261 (1976).

[28] P. R. Callis, T. W. Scott, and A. C. Albrecht, J. Phys. Chem. 78, 16 (1983); R. P. Ravaand and L. Goodman, J. Am. Chem. Soc. 104, 3815 (1982); B. Dick and G. Hohlneicher, Chem. Phys. Lett. 97, 324 (1983).

[29] R. A. Y. Jones, Physical and Mechanistic Organic Chemistry (Cambridge University Press, Cambridge, 1979).

[30] M. Graf and P. Vogl, Phys. Rev B 51, 4940 (1995).

[31] Otherwise one should use $U(t)=\Pi_{j} U\left(t_{j}+\Delta t, t_{j}\right)$, with $\Delta t$ small enough to ensure a correct chronological order of the infinitesimal time-evolution operators. Alternatively, one could employ the Magnus expansion of the time evolution operator (see, e.g., Ref. [32]).

[32] D. J. Tannor, Introduction to Quantum Mechanics: A TimeDependent Perspective (University Science Books, Sausalito, 2006).
[33] C. A. Coulson and G. S. Rushbrooke, Proc. Camb. Phil. Soc. 36, 193 (1940).

[34] J. A. Pople, Trans. Faraday Soc. 49, 1375 (1953).

[35] J. Čížek, J. Paldus, and I. Hubač, Int. J. Quantum Chem. 8, 951 (1974).

[36] J. Koutecký, J. Paldus, and J. Čížek, J. Chem. Phys. 83, 1722 (1985).

[37] J. Hubbard, Proc. R. Soc. Lond. A 276, 238 (1963).

[38] M. Büttiker, Y. Imry, and M. Y. Azbel, Phys. Rev. A 30, 1982 (1984).

[39] For a $k$-sites polygon $\Psi_{m}$ has $k$ components $e^{i(m+\phi) \theta}$, where $\theta=(2 \pi / k) q$ with $q=0, \pm 1, \pm 2, \ldots, \pm(k-1) / 2$ for odd $k$ and $q=0, \pm 1, \pm 2, \ldots \pm k / 2$ for even $k$. Then as $k$ rises up to infinity, $\theta$ becomes a continuous variable, and we end up with the eigenfunctions of a particle in a ring.

[40] A loosely related connection between alternance and timereversal symmetry is discussed in A. Laforgue, J. Čížek, and J. Paldus, J. Chem. Phys. 59, 2560 (1973).

[41] In molecules the one-site repulsion $U$ is generally estimated by means of the I-A. approximation, yielding $U=10.84 \mathrm{eV}$. The hopping parameter employed $-2.29 \mathrm{eV}$ is the so-called spectroscopic. See, e.g., R. G. Parr, The Quantum Theory of Molecular Electronic Structure (Benjamin, New York, 1963).

[42] G. V. Nazin, X. H. Qiu, and W. Ho, Science 302, 77 (2003).

[43] M. L. Mueller, X. Yan, J. A. McGuire, and L. S. Li, Nano Lett. 10, 2679 (2010).

[44] K. Kawasumi, Q. Zhang, Y. Segawa, L. T. Scott, and K. Itami, Nat. Chem. 5, 739 (2013).

[45] A. Streitwieser, Molecular Orbital Theory for Organic Chemists (Wiley, New York, 1961).

[46] Z. Y. Zeng, F. Claro, and A. Pérez, Phys. Rev. B 65, 085308 (2002).

[47] G. Klimeck, G. Chen, and S. Datta, Phys. Rev. B 50, 2316 (1994). 